\title{
Do the flash-lag effect and representational momentum involve similar extrapolations?
}

\author{
Timothy L. Hubbard* \\ Department of Psychology, Texas Christian University, Fort Worth, TX, USA
}

\section{Edited by:}

Yuki Yamada, Yamaguchi University, Japan

\section{Reviewed by:}

Gerrit W. Maus, University of California at Berkeley, USA

Jochen Musseler, RWTH Aachen

University, Germany

\section{${ }^{*}$ Correspondence:}

Timothy L. Hubbard, Department of Psychology, Texas Christian University, 2800 S. University, Fort Worth, TX 76129, USA. e-mail: timothyleehubbard@ gmail.com
In the flash-lag effect (FLE) and in representational momentum (RM), the represented position of a moving target is displaced in the direction of motion. Effects of numerous variables on the FLE and on RM are briefly considered. In many cases, variables appear to have the same effect on the FLE and on RM, and this is consistent with a hypothesis that displacements in the FLE and in RM result from overlapping or similar mechanisms. In other cases, variables initially appear to have different effects on the FLE and on RM, but accounts reconciling those apparent differences with a hypothesis of overlapping or similar mechanisms are suggested. Given that RM is simpler and accounts for a wider range of findings (i.e., RM involves a single stimulus rather than the relationship between two stimuli, RM accounts for displacement in absolute position of a single stimulus and for differences in relative position of two stimuli), it is suggested that (at least some cases of) the FLE might be a special case of RM in which the position of the target is assessed relative to the position of another stimulus (i.e., the flashed object) rather than relative to the actual position of the target.

Keywords: flash-lag effect, representational momentum, displacement, spatial mislocalization, spatial cognition
If observers view a moving target and a flashed (i.e., briefly presented) stationary object is presented in alignment with that moving target, the flashed object appears to lag behind the moving target. This is referred to as the flash-lag effect (FLE; Nijhawan, 1994; for review, Maus et al., 2010; Hubbard, in press-a). If observers view a moving target, the remembered final position of that target is shifted in the direction of target motion. This is referred to as representational momentum (RM; Freyd and Finke, 1984; for review, Hubbard, 2005, 2010). In the FLE (e.g., Shi and de'Sperati, 2008) and in RM (e.g., Hubbard, 1990), the represented position of a moving target is displaced forward, and the FLE (Nijhawan, 1994, 2008) and RM (Finke et al., 1986; Hubbard, 2005) have each been suggested to reflect compensation for delays in neural processing times and adaptation for realtime interaction with environmental stimuli. Surprisingly, there has been little comparison of the FLE and RM. Apparent similarities and differences of the FLE and RM are considered here, and it is suggested that displacement of the moving target in the FLE or in RM involves overlapping or similar mechanisms, or more radically, that the FLE is a special case of RM in which the represented position of the moving target is assessed relative to another object rather than relative to the actual target position.

\section{APPARENT SIMILARITIES OF THE FLE AND RM}

There are numerous apparent similarities of the FLE and RM. The existence of such similarities is consistent with a hypothesis that the FLE and RM result from overlapping or similar mechanisms.

\section{PERCEPTUAL SIMILARITIES}

Perceptual similarities involve effects of (1) velocity, (2) visual field, (3) a reference point, (4) multiple modalities, and (5) crossmodal information.

\section{Velocity}

The FLE (Nijhawan, 1994; Brenner and Smeets, 2000; LópezMoliner and Linares, 2006; Cantor and Schor, 2007; Wojtach et al., 2008) and RM (Hubbard and Bharucha, 1988; Hubbard, 1990) increase with increases in velocity of the moving target in the picture plane. The FLE (Lee et al., 2008) and RM (Hubbard, 1996) increase with increases in velocity of the moving target in depth. The FLE (Whitney et al., 2000) and RM (Finke et al., 1986) increase or decrease with acceleration or deceleration, respectively, of the moving target in the picture plane. The velocity effect is one of the most replicated effects in the FLE or RM literatures.

\section{Visual field}

Whether the moving target is in the left or right visual field does not consistently influence the FLE or RM, but if an effect of visual field occurs, the FLE (Kanai et al., 2004) and RM (Halpern and Kelly, 1993; White et al., 1993) are larger if the moving target is in the left visual field. Maus and Nijhawan (2009) presented variations of a horizontally moving FLE stimulus and reported a slightly greater effect of velocity on displacement of moving targets in the upper visual field, and Hubbard (2001) reported RM was larger for vertically moving targets in the lower than in the upper visual field. 


\section{Reference point}

$\mathrm{RM}$ is larger if a target moves toward rather than away from a landmark, and Hubbard and Ruppel (1999) suggested RM combined with a landmark attraction effect: If RM and landmark attraction operate in the same direction (motion toward a landmark), they sum and displacement is larger, whereas if RM and landmark attraction operate in opposite directions (motion away from a landmark), they partially cancel and displacement is smaller. Similarly, the FLE is larger if a target moves toward rather than away from the fixated region (Mateeff and Hohnsbein, 1988; Shi and Nijhawan, 2008), and Brenner et al. (2006) suggested the FLE combined with a bias toward the fixated region: If the FLE and bias toward fixation operate in the same direction (motion toward fixation), they sum and target displacement is larger, whereas if the FLE and bias toward fixation operate in opposite directions (motion away from fixation), they partially cancel and target displacement is smaller.

\section{Multiple modalities}

Most research on the FLE and RM presented visual stimuli. However, auditory stimuli can produce a FLE (Alais and Burr, 2003; Arrighi et al., 2005) and RM (Johnston and Jones, 2006), and haptic stimuli can produce a FLE (Nijhawan and Kirschfeld, 2003) and RM (Brouwer et al., 2005). It is possible that separate modality-specific mechanisms for the FLE and RM exist, but it is more parsimonious to posit a single mechanism or small number of higher-level mechanisms produces displacement of the moving target in the anticipated direction across multiple modalities (e.g., in higher-level processes or by top-down modulation of lower-level processes, Hubbard, 2005, 2006).

\section{Crossmodal information}

Visual information can influence the auditory FLE (Alais and Burr, 2003) and auditory RM (Hubbard and Courtney, 2010), and auditory information can influence the visual FLE (Vroomen and de Gelder, 2004) and visual RM (Teramoto et al., 2010). Kinesthetic information can influence the visual FLE (Cai et al., 2000; Schlag et al., 2000). Such influences of crossmodal information on the FLE and on RM are not consistent with solely lower-level explanations of the FLE or RM.

\section{COGNITIVE SIMILARITIES}

Cognitive similarities involve effects of (1) attention and cueing, (2) conceptual knowledge of target identity, (3) control and movement planning, (4) attribution regarding the source of motion, (5) frame of reference, and (6) neural mechanisms.

\section{Attention and cueing}

The FLE (Sarich et al., 2007) and RM (Hayes and Freyd, 2002) increase if attention is divided between the moving target and a concurrent irrelevant stimulus. If the position of the flashed object or moving target is cued, valid cues result in a smaller FLE (Brenner and Smeets, 2000; Namba and Baldo, 2004; Shioiri et al., 2010; but see Khurana et al., 2000) and smaller RM (Hubbard et al., 2009) than do invalid cues. RM is influenced by verbal cues given prior to target presentation (Hubbard, 1994), but whether the FLE is influenced by verbal cues has not been reported. The FLE (Maiche et al., 2007) and RM (Hubbard and Ruppel, 1999) increase if the target moves toward another object, and this might reflect spatial distribution of attention.

\section{Conceptual knowledge of target identity}

The FLE (Noguchi and Kakigi, 2008; Nagai et al., 2010) and RM (Reed and Vinson, 1996; Vinson and Reed, 2002) can be increased or decreased by conceptual knowledge regarding the identity of the moving target. Such influences suggest the FLE and RM do not result solely from lower-level processes, but rather result from higher-level processes or top-down modulation of lower-level processes. Also, the FLE (Moore and Enns, 2004) and RM (Kelly and Freyd, 1987) are diminished if the moving target does not maintain a consistent identity.

\section{Control and movement planning}

The FLE is decreased if participants control presentation of the flashed object (López-Moliner and Linares, 2006), and decreased or increased if participants control target motion with a computer mouse (Ichikawa and Masakura, 2006, 2010) or robotic arm (Scocchia et al., 2009), respectively. RM is decreased if participants control the moving target (Jordan and Knoblich, 2004; Stork and Müsseler, 2004). If participants judge the position of a moving target after acquiring experience controlling target motion, RM is larger (Jordan and Hunsinger, 2008); this was attributed to effects of action planning, and such effects might similarly account for the larger FLE when participants controlled the target in Scocchia et al. The FLE (Nijhawan, 1994, 2008) and RM (Finke et al., 1986; Hubbard, 2005) aid in planning body movements and in interactions with environmental stimuli.

\section{Attribution regarding the source of motion}

The FLE is influenced by whether participants believe they control target motion (Ichikawa and Masakura, 2006, 2010), and RM is influenced by whether participants attribute target motion to contact from an external stimulus or to an internal source (Hubbard, in press-b; Hubbard et al., 2001). Thus, the FLE and RM are decreased if motion is attributed to a source other than the target (e.g., the participant in the FLE, contact from another stimulus in RM), and this might result from higher-level processes or top-down modulation of lower-level processes.

\section{Frame of reference}

Studies of the FLE usually involve judgment of relative position (but see Munger and Owens, 2004; Shi and de'Sperati, 2008; Becker et al., 2009), whereas studies of RM usually involve judgment of absolute position; however, regardless of whether relative or absolute position is judged, represented target position in the FLE and in RM is displaced in the direction of target motion. The FLE (Maiche et al., 2007) and RM are influenced by whether another stimulus provides a landmark (Hubbard and Ruppel, 1999) or surrounding context (Hubbard, 1993), and localization of the flashed object in the FLE (van Beers et al., 2001) and moving target in RM (Hubbard, 1990, 
1997) are influenced by the direction of implied gravitational attraction $^{1}$.

\section{Neural mechanisms}

The FLE (Maus et al., 2013) and RM (Senior et al., 2002) are disrupted by transcranial magnetic stimulation of area MT. Kimura et al. (2011) suggested visual mismatch negativity might be related to the FLE and to RM. RM activates prefrontal cortex and anterior cingulate cortex (Rao et al., 2004); surprisingly, imaging information on the FLE has not been reported (although see Nijhawan, 2008, for discussion of potentially relevant neural mechanisms). The retina appears to compute a "crude extrapolation of the object's trajectory" (Gollisch and Meister, 2010, p. 155), and this is consistent with the FLE and with RM.

\section{APPARENT DIFFERENCES OF THE FLE AND RM}

There are fewer apparent differences than apparent similarities regarding the FLE and RM. In many cases, differences that initially appear inconsistent with a hypothesis of overlapping or similar mechanisms in the FLE and RM can be reconciled with that hypothesis.

\section{PERCEPTUAL DIFFERENCES}

Perceptual differences involve effects of (1) oculomotor behavior, (2) environment-centered direction, (3) object-centered direction, and (4) location within the target trajectory.

\section{Oculomotor behavior}

The FLE (Nijhawan, 2001) and RM (Kerzel, 2000) for a continuously moving target are decreased and increased, respectively, if participants use smooth pursuit eye movements to track that target. However, such a difference does not rule out overlapping or similar higher-level mechanisms for the FLE and RM any more than differences in oculomotor behavior with continuous motion, implied motion, or frozen-action photographs rule out overlapping or similar higher-level mechanisms for RM (for discussion, Hubbard, 2005, 2006, 2010). Oculomotor behavior modulates target displacement for only some types of visual stimuli $^{2}$, and so cannot be the sole cause of the FLE and RM

\footnotetext{
${ }^{1}$ An influence of direction of implied gravitational attraction on represented position is referred to as representational gravity (RG; Hubbard, 1995, 1997). van Beers et al. (2001) reported flashed objects located above or below the trajectory of a horizontally moving target were displaced away from the trajectory, and this displacement was larger if flashed objects were below the trajectory. Although RG was not considered by van Beers et al., the data they reported are consistent with a combination of RG and a bias away from the trajectory: If RG and bias away from the trajectory operate in the same direction (flashed object below the trajectory), they sum and displacement is larger, whereas if RG and bias away from the trajectory operate in opposite directions (flashed object above the trajectory), they partially cancel and displacement is smaller. Similarly, Hubbard $(1990,1997)$ reported that horizontally moving targets were displaced downward and forward (RG and RM operate in orthogonal directions) and that forward displacement was larger for descending targets (RG and RM operate in the same direction and sum) than for ascending targets (RG and RM operate in opposite directions and partially cancel).

${ }^{2}$ The FLE (e.g., Rizk et al., 2009) and RM (e.g., Munger et al., 1999) also occur with implied motion (referred to as station to station motion in the FLE literature), and RM also occurs for frozen-action photographs (e.g., Futterweit and Beilin, 1994); neither implied motion nor frozen-action photographs evoke
}

with visual stimuli. Consistent with this, the FLE and RM occur with auditory, haptic, and crossmodal stimuli, and the FLE and RM are influenced by higher-level processes (Hubbard, 2005, in press-a).

\section{Environment-centered direction}

The FLE is not influenced by whether targets descend or ascend (Ichikawa and Masakura, 2006, 2010), but RM is larger when targets descend than when targets ascend (Hubbard, 1990, 1997). Such findings would be consistent with a hypothesis of overlapping or similar mechanisms if the absolute positions of the moving target and the flashed object in the FLE were displaced forward equal distances (preserving their relative positions, cf. Hubbard, 2008), and these displacements were larger for ascending than for descending motion; however, measures of relative position typically used to study the FLE are not sensitive to displacement in absolute position.

\section{Object-centered direction}

The FLE (Nagai et al., 2010) and RM (Nagai and Yagi, 2001) are smaller and larger, respectively, if a target moves forward (its typical direction of motion) rather than backward (opposite its typical direction of motion). Such findings would be consistent with a hypothesis of overlapping or similar mechanisms if the (1) flashed object and moving target were displaced in the direction of motion, (2) displacement of the flashed object and of the moving target were smaller for backward than for forward motion, and (3) decrease in displacement with backward motion was larger for the flashed object than for the moving target. The difference between the moving target and the flashed object would appear larger for backward than for forward motion, and the FLE (difference in relative positions) would look larger even though absolute displacement was smaller.

\section{Location within the target trajectory}

A FLE usually occurs if the flashed object is presented at the beginning or midpoint, but not at the end, of the target trajectory (Hubbard, in press-a); however, RM is measured at the end of the target trajectory (Hubbard, 2005) ${ }^{3}$. One possibility consistent with a hypothesis of overlapping or similar mechanisms is that at the end of the trajectory, simultaneous decay of displacement of the moving target and displacement of the flashed object preserves their relative positions, resulting in no FLE, whereas at the

smooth pursuit eye movements. Oculomotor behavior involves the hardware implementation level or perhaps algorithmic level, whereas RM or the FLE might be caused by a higher-level mechanism involving the computational level (for discussion, Hubbard, 2005, 2006). Moreover, even if oculomotor behavior is correlated with extrapolation, such a linkage is not evidence that oculomotor behavior is causal in generation of extrapolation.

${ }^{3}$ A related forward displacement of target position at the beginning of the target trajectory is referred to as the Fröhlich effect (for review, Kerzel, 2010), but the relationship of the Fröhlich effect with the FLE or with RM is beyond the scope of this paper. Even so, it should be noted that just as the FLE might reflect RM in which the perceived final position of the moving target is assessed relative to an external stimulus (i.e., the flashed object) rather than relative to the actual final position of the target, a FLE in a flash-initiated display might reflect a Fröhlich effect in which the perceived initial position of the moving target is assessed relative to an external stimulus (i.e., the flashed object) rather than relative to the actual initial position of the target (for discussion, Hubbard, in press-a). 
beginning or midpoint of the trajectory, decay of displacement of the flashed object, coupled with continuing RM for the stillmoving target, results in a FLE. Alternatively, a flashed object near the end of the target trajectory might eliminate RM (Müsseler et al., 2002) or exhibit displacement similar to that of the target (Hubbard, 2008), and thus preserve the relative positions of the flashed object and moving target, resulting in no FLE.

\section{COGNITIVE DIFFERENCES}

Cognitive differences involve effects of (1) level of processing, (2) predictability, and (3) expertise.

\section{Level of processing}

In the FLE, the moving target and flashed object are perceptually available during judgment, and the FLE is usually considered a lower-level perceptual phenomenon. In RM, the moving target vanishes before judgment, and RM is usually considered a higher-level cognitive phenomenon. However, higher-level cognitive variables influence the FLE (Noguchi and Kakigi, 2008; Nagai et al., 2010), and lower-level perceptual variables influence RM (Kerzel et al., 2001; Kerzel, 2002a); thus, the FLE (Hubbard, in press-a) and RM (Hubbard, 2005) each involve lower-level perceptual processes and higher-level cognitive processes. In the FLE and in RM, represented target position at the sampled time (when the flashed object appeared or moving target vanished, respectively) is displaced forward, and this might involve overlapping or similar mechanisms at a lower or higher level.

\section{Predictability}

Forward displacement of a moving target in the FLE (Munger and Owens, 2004; Shi and de'Sperati, 2008) and RM (Finke and Freyd, 1985; Hubbard, 1990) suggests the FLE (Nijhawan, 1994, 2008) and RM (Hubbard, 1995, 2005) reflect predictions regarding subsequent target position ${ }^{4}$. Similarities in effects of attention and cueing in the FLE and in RM noted earlier suggest effects of predictability should be similar in the FLE and in RM. The FLE increases if predictability of the flashed object decreases (Baldo and Namba, 2002; Vreven and Verghese, 2005). However, RM decreases or increases if predictability (certainty) regarding target position is decreased by blocking target direction (Kerzel, 2002b) or increasing target blurriness (Fu et al., 2001), respectively. Manipulation of predictability in the FLE usually involves the flashed object, whereas manipulation of predictability in RM involves the moving target; it is not clear how predictability of a flashed object would influence localization of a moving target.

\footnotetext{
${ }^{4}$ It should be noted that there are at least two different senses of "predict" in the displacement literature. Munger and Minchew (2002) use "predict" to refer to an explicit and deliberate judgment regarding the subsequent potential position of the target and use "remember" to refer to judgments of the final position (see also Finke and Freyd, 1985), whereas Nijhawan (2008) uses "predict" to refer to an implicit and automatic process that extrapolates the representation. In the current paper, "predict" and "predictability" are used in the sense of an implicit and automatic extrapolation.
}

\section{Expertise}

RM is increased for stimuli in a domain of expertise (Blättler et al., 2010, 2011). The FLE in a given domain might be compensated for by experts in that domain (Catteeuw et al., 2009), and this suggests the FLE is decreased for stimuli in a domain of expertise. Compensation for the FLE could involve smaller displacement of the moving target or larger displacement of the flashed object. Only in the former case would effects of expertise differ for FLE and RM; the latter case is consistent with effects of RM on a nearby stationary object (Hubbard, 2008) and effects of expertise on RM for a moving target.

\section{CONCLUSIONS}

The FLE and RM involve forward displacement of the represented position of a moving target. A large group of variables have similar influences on the FLE and on RM, and this suggests the FLE and RM might arise from overlapping or similar mechanisms. A smaller group of variables appear to have dissimilar influences on the FLE and on RM, and potential ways to reconcile those differences with a hypothesis of overlapping or similar mechanisms were suggested. Interestingly, if perceived target position is assessed relative to the position of another stimulus, then displacement is usually referred to as a "flash-lag effect," whereas if perceived target position is assessed relative to the actual target position, then displacement is usually referred to as "representational momentum." This suggests the FLE is a special case of RM in which displacement of the moving target is assessed relative to the position of a flashed object rather than relative to the actual target position. A hypothesis that the FLE arises from overlapping or similar mechanisms as RM, or is a special case of RM, provides important constraints for theories of the FLE and of RM. Such a hypothesis also has heuristic value, as other variables that influence the FLE or RM (e.g., contrast, presence of feedback) could be predicted to have similar effects on RM and the FLE, respectively.

In defense of a mental extrapolation theory of the FLE, Nijhawan et al. (2004, p. 278) stated "a newer interpretation of a given phenomenon can be accepted over and above an existing one only if the newer interpretation is conceptually simpler (requires fewer assumptions) and/or is capable of explaining a wider class of empirical findings." By these criteria, an explanation of (at least some examples of) the FLE as a special case of RM should be preferred: RM is simpler than the FLE (e.g., RM involves one stimulus rather than the relationship between two stimuli) and accounts for a wider range of findings (e.g., involving a single stimulus as well as involving two stimuli). Indeed, the term "representational momentum" has a longer history in referring to extrapolation of a moving target, and so it might be appropriate and more parsimonious to consider this term and mechanism when referring to automatic extrapolation of target position, regardless of whether that extrapolation is measured relative to the position of another object or relative to the actual target position. Such an approach is consistent with models of spatial representation that address the FLE and RM (e.g., Müsseler et al., 2002; Jancke and Erlhagen, 2010) and suggests the possibility of overlapping or similar mechanisms of extrapolation. 


\section{REFERENCES}

Alais, D., and Burr, D. (2003). The "flash-lag" effect occurs in audition and cross-modally. Curr. Biol. 13, 59-63.

Arrighi, R., Alais, D., and Burr, D. (2005). Neural latencies do not explain the auditory and audiovisual flash-lag effect. Vision Res. 45, 2917-2925.

Baldo, M. V. C., and Namba, J. (2002). The attentional modulation of the flash-lag effect. Braz. J. Med. Biol. Res. 35, 969-972.

Becker, S. I., Ansorge, U., and Turatto, M. (2009). Saccades reveal that allocentric coding of the moving object causes mislocalization in the flashlag effect. Atten. Percept. Psychophys. 71, 1313-1324.

Blättler, C., Ferrari, V., Didierjean, A., and Marmèche, E. (2011). Representational momentum in aviation. J. Exp. Psychol. Hum. Percept. Perform. 37, 1569-1577.

Blättler, C., Ferrari, V., Didierjean, A., van Elslande, P., and Marmèche, E. (2010). Can expertise modulate representational momentum? Vis. Cogn. 18, 1253-1273.

Brenner, E., and Smeets, J. B. J. (2000). Motion extrapolation is not responsible for the flash-lag effect. Vision Res. 40, 1645-1648.

Brenner, E., van Beers, R. J., Rotman, G., and Smeets, J. B. J. (2006). The role of uncertainty in the systematic spatial mislocalization of moving objects. J. Exp. Psychol. Hum. Percept. Perform. 32, 811-825.

Brouwer, A. M., Thornton, I. M., and Franz, V. H. (2005). Forward displacement in grasping and visually judging pliers. Vis. Cogn. 12, 800-816.

Cai, R. H., Jacobson, K., Baloh, R., Schlag-Rey, M., and Schlag, J. (2000). Vestibular signals can distort the perceived spatial relationship of retinal stimuli. Exp. Brain Res. 135, 275-278.

Cantor, C. R. L., and Schor, C. M. (2007). Stimulus dependence of the flash-lag effect. Vision Res. 47, 2841-2854.

Catteeuw, P., Helsen, W., Gilis, B., van Roie, E., and Wagemans, J. (2009). Visual scan patterns and decisionmaking skills of expert assistant referees in offside situations. J. Sport Exerc. Psychol. 31, 786-797.

Finke, R. A., and Freyd, J. J. (1985). Transformations of visual memory induced by implied motions of pattern elements. J. Exp. Psychol. Learn. Mem. Cogn. 11, 780-794.

Finke, R. A., Freyd, J. J., and Shyi, G. C. W. (1986). Implied velocity and acceleration induce transformations of visual memory. J. Exp. Psychol. Gen. 115, 175-188.

Freyd, J. J., and Finke, R. A. (1984). Representational momentum. J. Exp. Psychol. Learn. Mem. Cogn. 10, 126-132.

Fu, Y. X., Shen, Y., and Dan, Y. (2001). Motion-induced perceptual extrapolation of blurred visual targets. J. Neurosci. 21, RC172.

Futterweit, L. R., and Beilin, H. (1994). Recognition memory for movement in photographs: a developmental study. J. Exp. Child Psychol. 57, 163-179.

Gollisch, T., and Meister, M. (2010). Eye smarter than scientists believed: neural computations in circuits of the retina. Neuron 65, 150-164.

Halpern, A. R., and Kelly, M. H. (1993). Memory biases in left versus right implied motion. J. Exp. Psychol. Learn. Mem. Cogn. 19, 471-484.

Hayes, A. E., and Freyd, J. J. (2002). Representational momentum when attention is divided. Vis. Cogn. 9, 8-27.

Hubbard, T. L. (1990). Cognitive representation of linear motion: possible direction and gravity effects in judged displacement. Mem. Cogn. 18, 299-309.

Hubbard, T. L. (1993). The effect of context on visual representational momentum. Mem. Cogn. 21, 103-114.

Hubbard, T. L. (1994). Judged displacement: a modular process? Am. J. Psychol. 107, 359-373.

Hubbard, T. L. (1995). Environmental invariants in the representation of motion: implied dynamics and representational momentum, gravity, friction, and centripetal force. Psychon. Bull. Rev. 2, 322-338.

Hubbard, T. L. (1996). Displacement in depth: representational momentum and boundary extension. Psychol. Res. 59, 33-47.

Hubbard, T. L. (1997). Target size and displacement along the axis of implied gravitational attraction: effects of implied weight and evidence of representational gravity. J. Exp. Psychol. Learn. Mem. Cogn. 23, 1484-1493.

Hubbard, T. L. (2001). The effect of height in the picture plane on the forward displacement of ascending and descending targets. Can. J. Exp. Psychol. 55, 325-330.

Hubbard, T. L. (2005). Representational momentum and related displacement in spatial memory: a review of the findings. Psychon. Bull. Rev. 12, 822-851.

Hubbard, T. L. (2006). Computational theory and cognition in representational momentum and related types of displacement: a reply to Kerzel. Psychon. Bull. Rev. 13, 174-177.

Hubbard, T. L. (2008) Representational momentum contributes to motion induced mislocalization of stationary objects. Vis. Cogn. 16, 44-67.

Hubbard, T. L. (2010). "Approaches to representational momentum: theories and models," in Space and Time in Perception and Action, eds R. Nijhawan and B. Khurana (New York, NY: Cambridge University Press), 338-365.

Hubbard, T. L. (in press-a). The flashlag effect and related mislocalizations: findings, properties, and theories. Psychol. Bull.

Hubbard, T. L. (in press-b). Launching, entraining, and representational momentum: evidence consistent with an impetus heuristic in perception of causality. Axiomathes.

Hubbard, T. L., and Bharucha, J. J. (1988). Judged displacement in apparent vertical and horizontal motion. Percept. Psychophys. 44, 211-221.

Hubbard, T. L., Blessum, J. A., and Ruppel, S. E. (2001). Representational momentum and Michotte's (1946/1963) "launching effect” paradigm. J. Exp. Psychol. Learn. Mem. Cogn. 27, 294-301.

Hubbard, T. L., and Courtney, J. R. (2010). Cross-modal influences on representational momentum and representational gravity. Perception 39, 851-862.

Hubbard, T. L., Kumar, A. M., and Carp, C. L. (2009). Effects of spatial cueing on representational momentum. J. Exp. Psychol. Learn. Mem. Cogn. 35, 666-677.

Hubbard, T. L., and Ruppel, S. E. (1999). Representational momentum and the landmark attraction effect. Can. J. Exp. Psychol. 53, 242-256.

Ichikawa, M., and Masakura, Y. (2006). Manual control of the visual stimulus reduces the flash-lag effect. Vision Res. 46, 2192-2203.

Ichikawa, M., and Masakura, Y. (2010). Reduction of the flash-lag effect in terms of active observation. Atten. Percept. Psychophys. 72, 1032-1044.

Jancke, D., and Erlhagen, W. (2010). "Bridging the gap: a model of common neural mechanisms underlying the Fröhlich effect, the flash-lag effect, and the representational momentum effect," in Space and Time in Perception and Action, eds R. Nijhawan and B. Khurana (New York, NY: Cambridge University Press), 422-440.
Johnston, H. M., and Jones, M. R. (2006). Higher order pattern structure influences auditory representational momentum. J. Exp. Psychol. Hum. Percept. Perform. 32, 2-17.

Jordan, J. S., and Hunsinger, M. (2008). Learned patterns of action-effect anticipation contribute to the spatial displacement of continuously moving stimuli. J. Exp. Psychol. Hum. Percept. Perform. 34, 113-124.

Jordan, J. S., and Knoblich, G. (2004). Spatial perception and control. Psychon. Bull. Rev. 11, 54-59.

Kanai, R., Sheth, B. R., and Shimojo, S. (2004). Stopping the motion and sleuthing the flash-lag effect: spatial uncertainty is the key to perceptual mislocalization. Vision Res. 44, 2605-2619.

Kelly, M. H., and Freyd, J. J. (1987). Explorations of representational momentum. Cogn. Psychol. 19, 369-401.

Kerzel, D. (2000). Eye movements and visible persistence explain the mislocalization of the final position of a moving target. Vision Res. 40, 3703-3715.

Kerzel, D. (2002a). The locus of "memory displacement" is at least partially perceptual: effects of velocity, expectation, friction, memory averaging, and weight. Percept. Psychophys. 64, 680-692.

Kerzel, D. (2002b). A matter of design: no representational momentum without predictability. Vis. $\operatorname{Cog} n$. 9, 66-80.

Kerzel, D. (2010). “The Fröhlich effect: past and present," in Space and Time in Perception and Action, eds R. Nijhawan and B. Khurana (New York, NY: Cambridge University Press), 321-337.

Kerzel, D., Jordan, J. S., and Müsseler, J. (2001). The role of perception in the mislocalization of the final position of a moving target. J. Exp. Psychol. Hum. Percept. Perform. 27, 829-840.

Khurana, B., Watanabe, K., and Nijhawan, R. (2000). The role of attention in motion extrapolation: are moving objects 'corrected' or flashed objects attentionally delayed? Perception 29, 675-692.

Kimura, M., Schröger, E., and Czigler, I. (2011). Visual mismatch negativity and its importance in visual cognitive sciences. Neuroreport 22 , 669-673.

Lee, T. C. P., Khuu, S. K., Li, W., and Hayes, A. (2008). Distortion in perceived image size accompanies flash lag in depth. J. Vis. 8:20. doi: 101167/8.11.20

López-Moliner, J., and Linares, D. (2006). The flash-lag is reduced when the flash is perceived as a 
sensory consequence of our action. Vision Res. 46, 2122-2129.

Maiche, A., Budelli, R., and GómezSena, L. (2007). Spatial facilitation is involved in flash-lag effect. Vision Res. 47, 1655-1661.

Mateeff, S., and Hohnsbein, J. (1988). Perceptual latencies are shorter for motion towards the fovea than for motion away. Vision Res. 28, 711-719.

Maus, G. W., Khurana, B., and Nijhawan, R. (2010). "History and theory of flash-lag: past, present, and future," in Space and Time in Perception and Action, eds R. Nijhawan and B. Khurana (New York, NY: Cambridge University Press), 477-499.

Maus, G. W., and Nijhawan, R. (2009). Going, going, gone: localizing abrupt offsets of moving objects. J. Exp. Psychol. Hum. Percept. Perform. 35, 611-626.

Maus, G. W., Ward, J., Nijhawan, R., and Whitney, D. (2013). The perceived position of moving objects: transcranial magnetic stimulation of area MT+ reduces the flash-lag effect. Cereb. Cortex 23, 241-247.

Moore, C. M., and Enns, J. T. (2004). Object updating and the flash-lag effect. Psychol. Sci. 15, 866-871.

Munger, M. P., and Minchew, J. H. (2002). Parallels between remembering and predicting an object's location. Vis. Cogn. 9, 177-194.

Munger, M. P., and Owens, T. R. (2004). Representational momentum and the flash-lag effect. Vis. Cogn. 11, 81-103.

Munger, M. P., Solberg, J. L., Horrocks, K. K., and Preston, A. S. (1999). Representational momentum for rotations in depth: effects of shading and axis. J. Exp. Psychol. Learn. Mem. Cogn. 25, 157-171.

Müsseler, J., Stork, S., and Kerzel, D. (2002). Comparing mislocalizations with moving stimuli: the Fröhlich effect, the flash-lag, and representational momentum. Vis. Cogn. 9, 120-138.

Nagai, M., Suganuma, M., Nijhawan, R., Freyd, J. J., Miller, G., and
Watanabe, K. (2010). "Conceptual influence on the flash-lag effect and representational momentum," in Space and Time in Perception and Action, eds R. Nijhawan and B. Khurana (New York, NY: Cambridge University Press), 366-378.

Nagai, M., and Yagi, A. (2001). The pointedness effect on representational momentum. Mem. Cogn. 29, 91-99.

Namba, J., and Baldo, M. V. C. (2004). The modulation of the flash-lag effect by voluntary attention. Perception 33, 621-631.

Nijhawan, R. (1994). Motion extrapolation in catching. Nature 370, 256-257.

Nijhawan, R. (2001). The flash-lag phenomenon: object motion and eye movements. Perception 30, 263-282.

Nijhawan, R. (2008). Visual prediction: psychophysics and neurophysiology of compensation for time delays. Behav. Brain Sci. 31, 179-239.

Nijhawan, R., and Kirschfeld, K. (2003). Analogous mechanisms compensate for neural delays in the sensory and the motor pathways; evidence from motor flash-lag. Curr. Biol. 13, 749-753.

Nijhawan, R., Watanabe, K., Khurana, B., and Shimojo, S. (2004). Compensation of neural delays in visual-motor behavior: no evidence for shorter afferent delays for visual motion. Vis. Cogn. 11, 275-298.

Noguchi, Y., and Kakigi, R. (2008). Knowledge-based correction of flash-lag illusion. J. Cogn. Neurosci. 20, 513-525.

Rao, H., Han, S., Jiang, Y., Xue, Y., Gu, H., Cui, Y., et al. (2004). Engagement of the prefrontal cortex in representational momentum: an fMRI study. Neuroimage 23, 98-103.

Reed, C. L., and Vinson, N. G. (1996). Conceptual effects on representational momentum. J. Exp. Psychol. Hum. Percept. Perform. 22, 839-850.

Rizk, J. K., Chappell, M., and Hine, T. J. (2009). Effect of motion smoothness on the flash-lag illusion. Vision Res. 49, 2201-2208.

Sarich, D., Chappell, M., and Burgess, C. (2007). Dividing attention in the flash-lag illusion. Vision Res. 47, 544-547.

Schlag, J., Cai, R. H., Dorfman, A. Mohempour, A., and Schlag-Rey, M. (2000). Extrapolating movement without retinal motion. Nature 403 38-39.

Scocchia, L., Actis-Grosso, R. de'Sperati, C., Stucchi, N., and Baud-Bovy, G. (2009). Observer's control of the moving stimulus increases the flash-lag effect. Vision Res. 49, 2363-2370.

Senior, C., Ward, J., and David, A. S. (2002). Representational momentum and the brain: an investigation into the functional necessity of V5/MT. Vis. Cogn. 9, 81-92.

Shi, Z., and de'Sperati, C. (2008) Motion-induced positional biases in the flash-lag configuration. Cogn. Neuropsychol. 25, 1027-1038.

Shi, Z., and Nijhawan, R. (2008). Behavioral significance of motion direction causes anisotropic flashlag, flash-drag, flash-repulsion, and movement-mislocalization effects. J. Vis.. 8:24. doi: 101167/8.7.24

Shioiri, S., Yamamoto, K., Oshida, H. Matsubara, K., and Yaguchi, H. (2010). Measuring attention using flash-lag effect. J. Vis. 10:10. doi: $10.1167 / 10.10 .10$

Stork, S., and Müsseler, J. (2004). Perceived locations and eye movements with action-generated and computer-generated vanishing points of moving stimuli. Vis. Cogn 11, 299-314.

Teramoto, W., Hidaka, S., Gyoba, J. and Suzuki, Y. (2010). Auditory temporal cues can modulate visual representational momentum. Atten. Percept. Psychophys. 72 2215-2226.

van Beers, R. J., Wolpert, D. M., and Haggard, P. (2001). Sensorimotor integration compensates for visual localization errors during smooth pursuit eye movements. J. Neurophysiol. 85, 1914-1922.

Vinson, N. G., and Reed, C. L. (2002). Sources of object-specific effects in representational momentum. Vis. Cogn. 9, 41-65.

Vreven, D., and Verghese, P. (2005). Predictability and the dynamics of position processing in the flash-lag effect. Perception 34, 31-44.

Vroomen, J., and de Gelder, B. (2004). Temporal ventriloquism: sound modulates the flash-lag effect. J. Exp. Psychol. Hum. Percept. Perform. 30, 513-518.

White, H., Minor, S. W., Merrell, J., and Smith, T. (1993). Representationalmomentum effects in the cerebral hemispheres. Brain Cogn. 22, 161-170.

Whitney, D., Murakami, I., and Cavanagh, P. (2000). Illusory spatial offset of a flash relative to a moving stimulus is caused by differential latencies for moving and flashed stimuli. Vision Res. 40, 137-149.

Wojtach, W. T., Sung, K., Truong, S., and Purves, D. (2008). An empirical explanation of the flash-lag effect. Proc. Natl. Acad. Sci. U.S.A. 105 16338-16343.

Conflict of Interest Statement: The author declares that the research was conducted in the absence of any commercial or financial relationships that could be construed as a potential conflict of interest.

Received: 11 February 2013; accepted: 05 May 2013; published online: 23 May 2013.

Citation: Hubbard TL (2013) Do the flash-lag effect and representational momentum involve similar extrapolations?. Front. Psychol. 4:290. doi: 10.3389/fpsyg.2013.00290

This article was submitted to Frontiers in Consciousness Research, a specialty of Frontiers in Psychology.

Copyright (c) 2013 Hubbard. This is an open-access article distributed under the terms of the Creative Commons Attribution License, which permits use, distribution and reproduction in other forums, provided the original authors and source are credited and subject to any copyright notices concerning any thirdparty graphics etc. 